\title{
A Generalized Kinetic Model for Compartmentalization of Organometallic Catalysis
}

\author{
Brandon J. Jolly ${ }^{1}$, Chong Liu ${ }^{1,2 *}$ \\ ${ }^{1}$ Department of Chemistry and Biochemistry, University of California, Los Angeles, California \\ 90095, United States \\ ${ }^{2}$ California NanoSystems Institute (CNSI), University of California, Los Angeles, Los Angeles, \\ CA 90095, USA
}

* To whom correspondence may be addressed. Email: chongliu@chem.ucla.edu 


\begin{abstract}
Compartmentalization is an attractive approach to enhance catalytic activity by retaining reactive intermediates and mitigating deactivating pathways. Such a concept has been well explored in biochemical and more recently, organometallic catalysis to ensure high reaction turnovers with minimal side reactions. However, a scarcity of theoretical framework towards confined organometallic chemistry impedes a broader utility for the implementation of compartmentalization. Herein, we report a general kinetic model and offer design guidance for a compartmentalized organometallic catalytic cycle. In comparison to a non-compartmentalized catalysis, compartmentalization is quantitatively shown to prevent the unwanted intermediate deactivation, boost the corresponding reaction efficiency $(\gamma)$, and subsequently increase catalytic turnover frequency (TOF). The key parameter in the model is the volumetric diffusive conductance $\left(F_{V}\right)$ that describes catalysts' diffusion propensity across a compartment's boundary. Optimal values of $F_{V}$ for a specific organometallic chemistry are needed to achieve maximal values of $\gamma$ and TOF. Our model suggests a tailored compartment design, including the use of nanomaterials, is needed to suit a specific organometallic catalysis. This work provides justification and design principles for further exploration into compartmentalizing organometallics to enhance catalytic performance.
\end{abstract}

KEYWORDS: Compartmentalization, organometallic catalysis, microscopic mass transport, nanomaterials 


\section{INTRODUCTION}

Compartmentalization has been well documented in biochemical literature as one method for achieving efficient in vivo tandem catalysis by encapsulating enzymes in well-defined microand nano-structures. ${ }^{1-7}$ By controlling the diffusion of species in and out of compartment boundaries, nature is able to retain reactive or toxic intermediates, increase local substrate concentration, and mitigate deactivating or competing pathways. ${ }^{1-7}$ For example, carboxysome microcompartments enhance the rate of $\mathrm{CO}_{2}$ fixation by encapsulating the cascade of carbonic anhydrase and ribose 1,5-bisphosphate carboxylase/oxygenase to generate high local concentration of $\mathrm{CO}_{2}$ and exclude deactivating $\mathrm{O}_{2}$ within their polyhedral structures. ${ }^{8,9}$ Also, the last two steps of tryptophan biosynthesis - the conversion of indole-3-glycerol-phosphate to indole and then to tryptophan - takes advantages of the substrate-channeling effect bestowed by compartmentalized subunits of tryptophan synthase. ${ }^{10,11}$ Here, a hydrophobic tunnel between the two subunits retains the indole intermediate, which prevents its free diffusion and participation in deactivating side reactions. ${ }^{10}$ With billions of years of evolution, compartmentalization appears the mainstay of biology to manage the complex network of biochemical reactions that are frequently competing and incompatible with each other in a homogenous solution.

The success of natural compartmentalized enzyme cascades inspires the development of bio-mimetic synthetic catalysis with organometallic chemistry being the latest frontier. Multiple groups have employed well-defined spatial organization at the nano- and microscopic levels to construct in vitro biocatalytic and organometallic cascades with enhanced catalytic performance. ${ }^{2}$, 3, 12-16 Encapsulating NiFe hydrogenase in virus capsids improves its proteolytic and thermal stability as well as enhances the rate of $\mathrm{H}_{2}$ production. ${ }^{12}$ Confining a biochemical cascade of $\beta$ galactose, glucose oxidase, and horse radish peroxidase in metal-organic frameworks led to an enhancement of reaction yield in comparison to a freely diffusing analogue. ${ }^{13,14}$ The extent to which reaction yields are enhanced in confined enzyme cascades is reported to correlate with the distance among active sites, suggesting that spatial organization or localization of catalysts is beneficial in tandem or cascade reactions. ${ }^{15}$ In addition to biocatalysis, recently compartmentalization of organometallic catalysts has been experimentally demonstrated. ${ }^{17-23}$ For example, our group employed a nanowire-array electrode to pair seemingly incompatible $\mathrm{CH}_{4}$ activation based on $\mathrm{O}_{2}$-sensitive rhodium (II) metalloradical ( $\mathrm{Rh}(\mathrm{II})$ ) with $\mathrm{O}_{2}$-based oxidation for 
$\mathrm{CH}_{3} \mathrm{OH}$ formation. ${ }^{17,} 24$ The application of a reducing potential to the nanowire array electrode created a steep $\mathrm{O}_{2}$ gradient within the wire array electrode, such that an anoxic region was established at the bottom of the wires. As a result, a catalytic cycle was formed in which the airsensitive $\mathrm{Rh}(\mathrm{II})$ activated $\mathrm{CH}_{4}$ in the $\mathrm{O}_{2}$-free region of the wire array electrode, while $\mathrm{CH}_{3} \mathrm{OH}$ synthesis proceeded in the aerobic domain. Moreover, by substituting a planar electrode (no anoxic region) for the nanowire array, $\mathrm{CH}$ activation and $\mathrm{CH}_{3} \mathrm{OH}$ generation are negligible. ${ }^{17}$ The retainment of the ephemeral $\mathrm{Rh}(\mathrm{II})$ intermediate by the nanowire electrode for catalytic $\mathrm{CH}_{4}$-to$\mathrm{CH}_{3} \mathrm{OH}$ conversion ${ }^{17}$, 24 encourages us to further explore the design principles of compartmentalizing organometallic cascades for higher turnovers with mitigated deactivation pathways.

We envision that a theoretical framework for organometallic catalysis will expand the use of compartmentalization for organometallic chemistry. In biochemistry, mathematical modeling of confined enzyme cascades has been well developed and offers the design principles in natural systems $^{11,25}$ and for engineered bio-compartments. ${ }^{11,16,25,26}$ The models pinpoint a key parameter, volumetric diffusive conductance $\left(F_{V}\right)$, which describes the diffusion propensity across a compartment's boundary. $F_{V}$ is determined by a compartment's surface-to-volume ratio and its boundary's permeability. ${ }^{26,27}$ An optimal value of $F_{V}$ tailored to the specific biochemical reactions are needed in order to achieve better reactivity in comparison to the non-compartmentalized alternative. Similarly, we note that further development of compartmentalized organometallic chemistry demands a quantitative design principle applicable towards a model catalytic cycle that includes oxidative addition (OA), isomerization/migratory insertion (Iso/MI), and reductive elimination (RE) along with undesirable deactivation pathways (Figure 1A). Yet there has been a paucity of theoretical treatment despite progress in experimental demonstration. ${ }^{17-23} 22$ Such a lack of theoretical treatment motivates us to establish a general kinetic model and quantitatively investigate how compartmentalization will affect the competing reaction pathways and the corresponding turnover of the desired organometallic catalysis.

Here we report a general kinetic model and offer design guidance for a compartmentalized organometallic catalytic cycle. We took advantage of the established theoretical frameworks in biochemistry ${ }^{16,25,26}$ and applied such kinetic frameworks to a model compartmentalized organometallics with competing deactivation pathways (Figure 1A), ${ }^{28}$ and an analogous noncompartmentalized cycle (Figure 1B). We examined three metrics in the catalytic cycle in Figure 
$1 \mathrm{C}: 1)$ reaction efficiency $(\gamma)$ that gauges the percentage of intermediates funneled towards desirable catalytic turnover over deactivation pathways, 2) the flux of catalytic intermediates out of the compartment to be deactivated $\left(R_{I}\right)$, and 3) turnover frequency (TOF) that measures the steady-state catalytic rate despite intermediate deactivation. A compartmentalized system can significantly outperform a homogeneous counterpart with respect to $\gamma$ and $T O F$ with a lower value of $R_{I}$, at $F_{V}$ values smaller than the intrinsic kinetics of the organometallic cycle in question. We showcased how the developed model can serves as a guiding design principle for specific organometallic catalysis for maximal $\gamma$ and TOF. The established kinetic model can be adapted to suit a plethora of catalytic cycles or materials-based compartments, offering a framework to be expanded on for advanced compartmentalization of chemical catalysis.

\section{RESULTS AND DISCUSSION}

\section{Establishing a general kinetic framework of compartmentalization for an organometallic catalytic cycle}

We modelled a three-step catalytic cycle consisting of oxidative addition (OA), isomerization/migratory insertion (Iso/MI), and reductive elimination (RE) steps in the context of a compartmentalized system with multiple deactivation pathways in the solution (Figure 1A). ${ }^{28}$ Within the model, a catalytic species $C a t$ of a constant total concentration $\left(C_{\text {cat }}\right)$ in the bulk may diffuse into the compartment of volume $V$ and bind substrate molecule $A$ through oxidative addition to form intermediate species $C a t-A$, either first $(\mathrm{m}=1)^{29-32}$ or pseudo-second-order (m $=2)^{24,33,34}$ with respect to $C a t$ (rate constant $k_{1}$ ). The yielded $C a t-A$ species can yield product adduct species $C a t-B$ through isomerization or migratory insertion (rate constant $k_{2}$ ). Lastly, the catalytic cycle is completed by the reductive elimination transforming Cat $-B$ back to Cat with the release of product $B$ (rate constant $k_{3}$ ). In addition to the catalytic cycle, for the sake of generalizability we presume that $C a t, C a t-A$ and $C a t-B$ intermediates can diffuse across the compartment and there are two possible competing deactivation pathways that exist in the homogenous solution outside the compartment. The deactivations of $C a t-A$ and $C a t-B$ are presumed pseudo-first-order with respect to the intermediates with rate constants $k_{e 2}$ and $k_{e 3}$,

respectively ( $k_{e 2}$ and $k_{e 3}$ are discussed in further detail in Supplementary Information Section 3). 
Such established model of catalytic cycle is generally applicable to a broad range of organometallic catalysis with concurrent deactivation issues.

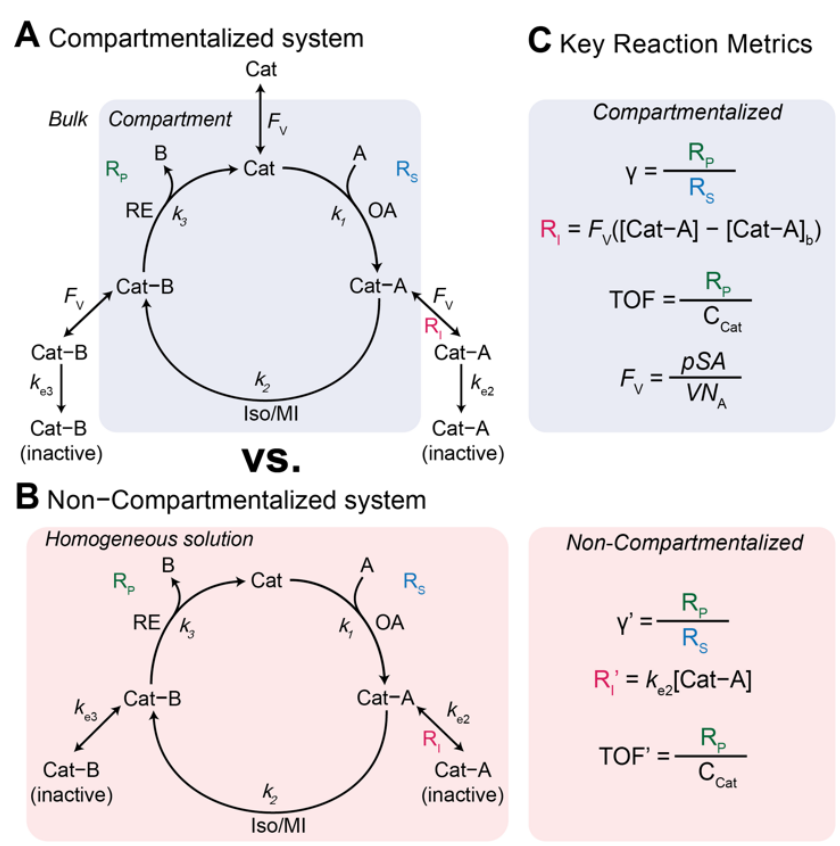

Figure 1. (A) Schematic of a general compartmentalized catalytic cycle in organometallic chemistry and (B) an analogous non-compartmentalized (freely diffusing) system. (C) Key reaction metrics used to assess and compare catalytic performance. $\gamma$ - reaction efficiency, $R_{S}$ - rate of substrate consumption, $R_{I}$ - rate of intermediate outflux/elimination, $R_{P}$ - rate of product formation, TOF - turnover frequency, $F_{V}$ - volumetric diffusive conductance, $p-$ compartment permeability, $S A$ - surface area, $V$ - compartment volume, $N_{A}$ - Avogadro's number, OA - oxidative addition (rate constant $k_{1}$ ), Iso/MI - isomerization/migratory insertion (rate constant $k_{2}$ competing deactivation rate constant $k_{e 2}$ ), $\mathrm{RE}$ - reductive elimination (rate constant $k_{3}$ competing deactivation rate constant $k_{e 3}$ ).

We assign volumetric diffusive conductance $\left(F_{V}\right)$ to quantitatively describe the extent of mass transport, predominantly diffusion-based, between the compartment and the surrounding bulk solution. As a measure of diffusion across the compartment's boundary, $F_{V}$ equals the product of compartment boundary's permeability $(p)$ and its total surface area $(S A)$, while normalized by the volume $(V)$ of the corresponding compartment and Avogadro's number $\left(N_{A}\right)$ (Figure $\left.1 \mathrm{C}\right) .{ }^{26} F_{V}$ describes a molecule's tendency to contribute a diffusion flux across the compartment under a given concentration difference across the compartment's boundary. As $p$ is linearly proportional to the species' diffusion coefficient $(D)$ and inversely proportional to the distance of diffusion path across the boundary, ${ }^{35} p, V$, and $S A$ depend on not only the compartment's geometric dimensions (for $p, V$, and $S A$ ) but also the materials' property of the compartment (for $p$ ). Since $F_{V}$ governs 
the rate of mass exchange in and out of a compartment, the design of compartment's geometric dimension, surface-to-volume ratio, and materials' property should have significant impacts on the overall catalytic turnover.

Additional assumptions were included in the analysis of the established model. First, we aim to study the steady-state phenomena of compartmentalized catalysis so we assume a constant, time-independent concentration of Cat, Cat - A and Cat - B in both the compartment ([Cat], $[\mathrm{Cat}-A]$, and $[\mathrm{Cat}-B]$, respectively) as well as the surrounding solution $\left([\mathrm{Cat}]_{b} \equiv C_{c a t}\right.$ (vide supra), $[\text { Cat }-A]_{b}$ and $[C a t-B]_{b}$, respectively). Similarly, in the bulk solution substrate $A$ is maintained at a constant concentration $\left(C_{A}\right)$ and fast removal of product $B$ is ensured $([B] \rightarrow 0)$. Such assumptions pertain to a flow reactor with sufficient amount of homogenous catalysts, presence of pre-catalysts or a batch reaction under high catalyst loading and low conversion. Alternatively, a constant total catalyst concentration $\left(C_{\text {Cat,total }}\right)$ including all catalytic species can be presumed (Supplementary Information Section 2). We note that this an alternative scenario,

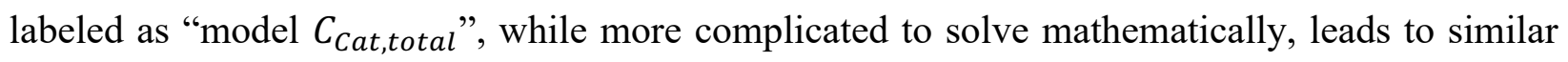
conclusions (Figures S5-7) and reinforces the general applicability of our results presented here. Second, the diffusion across the compartment boundary is considered for Cat, Cat - A and Cat $B$ with same values of $F_{V}$, given the fact that the catalytic center are frequently more bulky in comparison to the substrate/product and the catalytic intermediates typically have similar diffusion coefficients despite the reaction-related adducts. This also implies that substrate $A$ and product $B$ are considered to diffuse fast enough with effectively minimal concentration gradients.

A set of steady-state kinetic equations are constructed to reflect both the compartmentalized and non-compartmentalized scenarios (equations S1-5 and S67-69) for an organometallic catalytic cycle following the analysis protocols established in biochemistry. ${ }^{26}$ Comparing to the non-compartmentalized case that only includes reactions in the homogenous solution (equation S67-69), the equations for the compartmentalized case (equation S1-5) additionally consider the reactions in the compartment as well as the mass transport across the boundary, whose magnitudes are governed by $F_{V}$ and the concentration gradients across the compartment's boundary. Detailed mathematical treatment of the established equations can be found in Supplementary Section 1 and a few key outputs of the model are evaluated here. As one of the proposed benefits of compartmentalization is the capability of retaining reactive 
intermediates within the compartment without significant deactivation, we are interested in evaluating the steady-state consumption rate of substrate $A(R s)$, the generation rate of product $\mathrm{B}$ $\left(R_{P}\right)$, and the deactivation rate of intermediates Cat $-A\left(R_{I}\right)$. Moreover, in both compartmentalized and non-compartmentalized scenarios, we aim to analyze the rate of reaction (in the form of TOF) and the efficacy of transforming the substrate $A$ into targeted product $B$ (in the form of $\gamma$ ) that is defined as the percentage of intermediates funneled towards desirable catalytic turnover. ${ }^{16,26}$ In both cases, $\gamma$ is calculated as the ratio between the formation rate of product $B$ and the consumption rate of substrate $A$. In the case of pseudo-first-order kinetics towards Cat in oxidative addition $(\mathrm{m}=1), \gamma, R_{I, m=1}$, and $T O F_{m=1}$ in a compartmentalized system can be expressed as,

$\gamma=\frac{k_{2} k_{3}}{\left(f_{2}+k_{2}\right)\left(f_{3}+k_{3}\right)}$

$R_{I, m=1}=\frac{k_{1} k_{e 2} F_{V}^{2} C_{C a t} C_{A}}{\left(a_{1}+F_{V}\right)\left(f_{2}+k_{2}\right)\left(F_{V}+k_{e 2}\right)}$

$T O F_{m=1}=\frac{k_{1} k_{2} k_{3} F_{V} C_{A}}{\left(a_{1}+F_{V}\right)\left(f_{2}+k_{2}\right)\left(f_{3}+k_{3}\right)}$

in which,

$f_{2}=\frac{F_{V} k_{e 2}}{F_{V}+k_{e 2}}$ and $f_{3}=\frac{F_{V} k_{e 3}}{F_{V}+k_{e 3}}$

$a_{1}=k_{1} C_{A}-\frac{k_{1} k_{2} k_{3} C_{A}}{\left(f_{2}+k_{2}\right)\left(f_{3}+k_{3}\right)}$

While the $\gamma, R_{I, m=1}$, and TOF in a non-compartmentalized scenario, denoted as $\gamma^{\prime}, R_{I, m=1}^{\prime}$, and $T O F_{m=1}^{\prime}$ are expressed as,

$\gamma^{\prime}=\frac{k_{2} k_{3}}{\left(k_{2}+k_{e 2}\right)\left(k_{3}+k_{e 3}\right)}$

$R_{I, m=1}^{\prime}=\frac{k_{1} k_{e 2} C_{C a t} C_{A}}{k_{2}+k_{e 2}}$

$T O F_{m=1}^{\prime}=\frac{k_{1} k_{2} k_{3} C_{A}}{\left(k_{2}+k_{e 2}\right)\left(k_{3}+k_{e 3}\right)}$

The derivation of $\gamma, R_{I}$, and TOF for pseudo-second-order kinetics towards Cat in oxidative addition $(\mathrm{m}=2)$ as well as the results of model $C_{\text {Cat,total }}$ (vide supra) can be found in Sections 1 
and 2 of the Supplementary Information, respectively. The mathematical expressions for $\gamma, R_{I}$, and TOF results under compartmentalized and non-compartmentalized scenarios with $\mathrm{m}=1,2$ are shown in Table S1-2 as a reference. The successful construction and derivation of a general kinetic model in organometallic catalysis warrants quantitative evaluation about efficacy of compartmentalization under different reaction kinetics and compartment properties.

\section{An exemplary numerical comparison between compartmentalized and non- compartmentalized catalysis}

The derived analytical solutions to the established kinetic model allow us to numerically calculate the values of $\gamma, R_{I}$, and TOF in both compartmentalized and non-compartmentalized scenarios. Specifically, we set out to evaluate when compartmentalization is beneficial under a given set of parameters pertaining to the compartment's property and kinetics of organometallic reactions. As an introductory example representative to a typical organometallic catalytic cycle, we assume that $C_{\text {cat }}=1 \mathrm{mM}$ and $C_{\mathrm{A}}=10 \mathrm{mM}$, as organometallic catalytic systems often operate near $10 \mathrm{~mol} \%$ catalyst loading. ${ }^{36}$ Values of kinetic parameters are $k_{1}=\in\left[10^{-5}, 10^{4}\right] \mathrm{M}^{-1} \bullet \mathrm{s}^{-1}$ for $\mathrm{m}=1^{29-32}$ and $k_{1}=\in\left[10^{-3}, 10^{6}\right] \mathrm{M}^{-2} \cdot \mathrm{s}^{-1}$ for $\mathrm{m}=2,{ }^{17,24,33,34} k_{2} \in\left[10^{-3}, 10^{6}\right] \mathrm{s}^{-1},{ }^{37,38}$ and $k_{3}=10^{6}$ $\mathrm{s}^{-1}$. When either $k_{1}$ or $k_{2}$ are not a variable of interest, they are set to average values of $k_{1}=0.1$ $\mathrm{M}^{-1} \cdot \mathrm{s}^{-1}$ for $\mathrm{m}=1^{29-32}$ and $10 \mathrm{M}^{-2} \cdot \mathrm{s}^{-1}$, and $k_{2}=10^{3} \mathrm{~s}^{-1}$ The selection of those kinetic parameters is based on reviews of oxidative addition and migratory insertion, as well as reported kinetic studies using techniques such as time resolved infrared spectroscopy for transient species on the intermediates during carbonylation and $\mathrm{O}_{2}$ reduction and transfer, among others. ${ }^{17,}$ 24, 29-34, 37, 38 Selection of $k_{3}$ parameter value implicitly assumes fast reductive elimination from $C a t-B$, which is supported by the observation that reductive eliminations are often not the rate determining step in a catalytic cycle. ${ }^{39,40}$ The values of deactivation kinetics $k_{e 2}$ for $C a t-A$ and $k_{e 3}$ for $C a t-B$ are selected with additional assumptions, given the dearth of reported kinetic values for the less exciting deactivation steps. As the reductive elimination from Cat $-B$ is sufficiently fast, our primary focus is to examine how the deactivation from $C a t-A$ hence the comparison between $k_{2}$ and $k_{e 2}$ will affect the overall catalysis. Subsequently we assign $k_{e 3}=k_{3}=1 \times 10^{6} \mathrm{~s}^{-1}$ so that the rate of competing deactivation from $C a t-B$ is no lower than rate of reductive elimination. Similarly, when $k_{e 2}$ is not a variable of interest, it is set $k_{e 2}=k_{2}=1 \times 10^{3} \mathrm{~s}^{-1}$ to match the kinetics 
of Iso/MI. Last, we set $F_{V} \in[30,600] \mathrm{s}^{-1}$, whose range is estimated based on the diffusion coefficient of $9 \times 10^{-10} \mathrm{~m}^{2} \bullet \mathrm{s}^{-1}$ from tabulated organometallic catalysts, ${ }^{41,42}$ as well as the geometry and properties of reported microscopic compartments including the use of nanowire array electrode in our previous work (see Supplementary Information Section 3). ${ }^{17-20,43-45}$ Overall, our selection of kinetic values here represents an organometallic catalytic cycle whose oxidative addition step is turnover-limiting and the deactivation of yielded Cat $-A$ intermediate is the most critical issue, while the fast reductive elimination leaves the deactivation of $C a t-B$ species secondary in terms of $\gamma$ and TOF. With varying values of $F_{V}$ and changing ratios between the values of $k_{2}$ and $k_{e 2}$, the trend of compartmentalization's efficacy can be unveiled.

The calculated values of $\gamma, R_{I}$, and TOF as a function of $k_{2}$ and $F_{V}$ illustrate that compartmentalization generally outperforms the non-compartmentalized scenarios with a higher tolerance towards undesirable deactivation reactions (Figure 2). Under a fixed rate constant of deactivation $\left(k_{e 2}=1 \times 10^{3} \mathrm{~s}^{-1}\right)$ and a pseudo-first-order oxidative addition $\left(k_{1}=0.1 \mathrm{M}^{-1} \cdot \mathrm{s}^{-1}\right.$ for $\mathrm{m}$ $=1$ ), values of $\gamma, R_{I}$, and TOF in a compartmentalized system are plotted as a function of both $k_{2}$ and $F_{V}$ in Figure 2A-C. The rate of oxidative addition $\left(k_{2}\right)$ is understandably a predominant factor in all three plots. When $k_{2}$ is much smaller than the rate of deactivation $\left(k_{e 2}\right), \gamma$ approaches zero (Figure 2A) when the deactivation of $C a t-A$ outcompetes the step of isomerization/migratory insertion, leading to higher rate of deactivation $\left(R_{\mathrm{I}}\right.$ in Figure $\left.2 \mathrm{~B}\right)$ and lower TOF value (Figure 2C); alternatively when $k_{2}$ is much larger than $k_{e 2}$ and the deactivation step is less relevant, $\gamma$ plateaus towards unity with concomitant increase in TOF. Despite the dominant role of $k_{2}$, whether or not the system is compartmentalized strongly affects the values of $\gamma, R_{I}$, and TOF (Figure 2D-F). While the trend is generally applicable for all values of $F_{V}$, a specific case when $F_{V}=320 \mathrm{~s}^{-1}$, corresponding to the nanowire array electrode for $\mathrm{CH}_{4}-$ to- $\mathrm{CH}_{3} \mathrm{OH}$ conversion in our previous work, ${ }^{17}$ illustrates under which situation the advantages of compartmentalization will be observed. As the value of $k_{2}$ increases, reaction efficiency $\gamma$ (red trace in Figure 2D) increases in a sigmoidal fashion when $k_{2}$ approaches the value of $F_{V}$ with compartmentalization, while $\gamma$ in a non-compartmentalized case (black trace in Figure 2D) won't increase until $k_{2}$ approaches the value of $k_{e 2}$. Similarly, with $F_{V} \ll k_{e 2}$ and under a reasonably large value of $k_{2}$, compartmentalization suppresses the rate of deactivation $\left(R_{I}\right.$, Figure $\left.2 \mathrm{E}\right)$ and increases the $T O F$ (Figure $2 \mathrm{~F}$ ) by roughly no less than one order of magnitude. Evaluations assuming a pseudo- 
second-order kinetic towards Cat in the step of oxidative addition $(\mathrm{m}=2)$ lead to the same conclusion (Supplementary Figures S3A and S4A) and reinforces the conclusion's general applicability. Those observations suggest that the strategy of compartmentalization allows a

A $\gamma\left(k_{2}, F_{v}\right)$

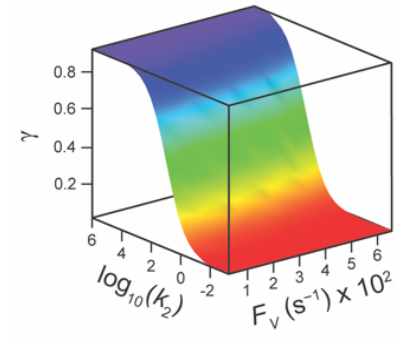

D $\gamma\left(k_{2}, F_{\mathrm{v}}=320 \mathrm{~s}^{-1}\right)$

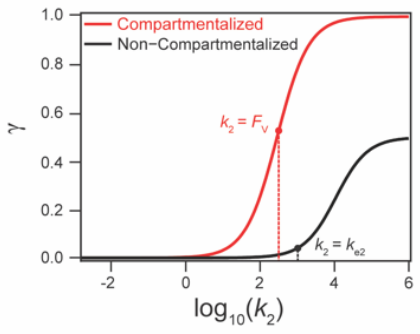

B $\log _{10}\left(R_{l, m=1}\left(k_{2}, F_{v}\right)\right)$

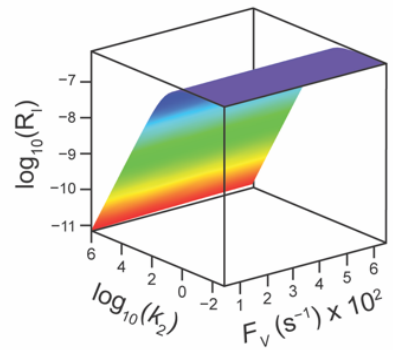

E $\log _{10}\left(R_{l, m=1}\left(k_{2}, F_{\mathrm{V}}=320 \mathrm{~s}^{-1}\right)\right)$

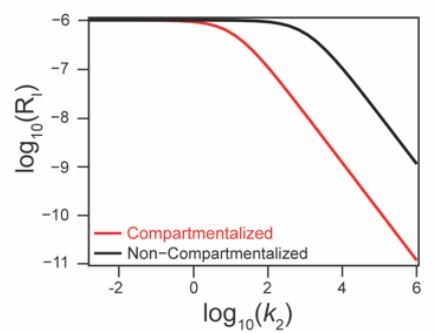

C $\log _{10}\left(\mathrm{TOF}_{\mathrm{m}=1}\left(k_{2}, F_{\mathrm{v}}\right)\right)$

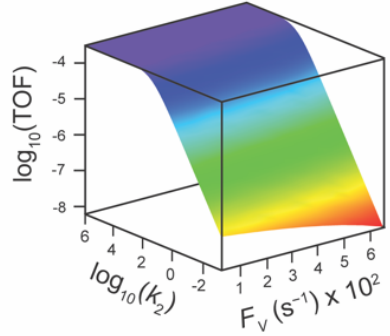

$\mathbf{F} \log _{10}\left(\operatorname{TOF}_{\mathrm{m}=1}\left(k_{2}, F_{\mathrm{v}}=320 \mathrm{~s}^{-1}\right)\right)$

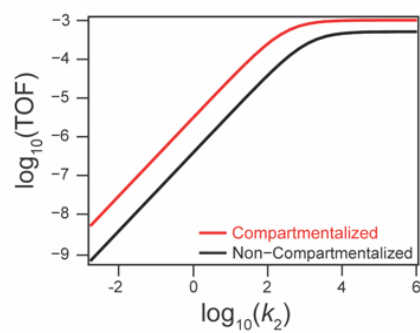

Figure 2. Graphical representations of compartmentalized reaction efficiency $(\gamma)$, rate of intermediate outflux $\left(R_{I}\right)$, and turnover frequency $(T O F)$ as a function of volumetric diffusive conductance $\left(F_{V}\right)$ and logarithm of the rate constant for isomerization or migratory insertion (Iso/MI) $\left(k_{2}\right)$ (A-C). Panels (D-F) comparison between compartmentalized (set to $F_{V}=320 \mathrm{~s}^{-1}$ ) and non-compartmentalized $\gamma, R_{I}$, and TOF. Kinetic parameters for panels $\mathbf{A}-\mathbf{F}$ are set at rate constant for oxidative addition $\left(k_{1}=0.1 \mathrm{M}^{-1} \mathrm{~s}^{-1}\right.$ ) (excluding $\gamma$ plots $\mathbf{A}$ and $\mathbf{D}$ ), rate constant for Cat $-A$ deactivation $\left(k_{e 2}\right)$ in competition with Iso/MI $\left(k_{e 2}=1 \times 10^{3} \mathrm{~s}^{-1}\right)$, and rate constant for reductive elimination $\left(k_{3}\right)$ and $C a t-B$ deactivation $\left(k_{e 3}\right)$ in competition $\left(k_{3}=k_{e 3}=1 \times 10^{6} \mathrm{~s}^{-1}\right)$, based on literature reports on the kinetics of relevant organometallic systems (vide supra).

catalytic cycle to be much more tolerant towards undesirable side reactions, as long as $F_{V}$ is much smaller than $k_{e 2}\left(F_{V} \ll k_{e 2}\right)$ with a judicious compartment design.

Additional examination suggests that a less "leaky" compartment with smaller $F_{V}$ value should be more effective. The difference of $\gamma$ values between compartmentalization and noncompartmentalization is the biggest when $F_{V}<k_{2} \ll k_{e 2}$ (Figure 2D). Such a trend is more apparent when $\gamma, R_{I}$, and TOF were plotted as a function of $F_{V}$ under fixed values of $k_{2}$ and $k_{e 2}$ (Figure $3 \mathrm{~A}-\mathrm{C}$ ). In both situations when $\mathrm{m}=1$ and $\mathrm{m}=2$, a larger value of $F_{V}$ leads to smaller values of $\gamma$ and TOF and large value of $R_{I}$. This suggests that a more "leaky" compartment is not sufficient to conserve the yielded intermediates and is more prone to deactivation. A similar 
conclusion can be obtained when investigating the dependence of $\gamma, R_{I}$, and TOF as a function of $F_{V}$ and $k_{e 2}$ (Figure 3D-F, and Figure S3B and S4B). Significant decrease of $\gamma$ and increase of $R_{I}$ was observed at high $F_{V}$ values, particularly at high $k_{e 2}$ values when deactivation is much faster and intermediate $C a t-A$ has a much shorter life time once it diffuses out of the compartment.

The above noted effects can be mathematically justified based on our derived equations. When the value of $F_{V}$ is similar to or even larger than $k_{e 2}$ or $k_{e 3}\left(F_{V} \gtrsim k_{e 2}\right.$ or $\left.k_{e 3}\right)$,

$\left.f_{2}\right|_{F_{V} \gtrsim k_{e 2}}=\frac{F_{V} k_{e 2}}{F_{V}+k_{e 2}}=k_{e 2} \quad$ and $\left.\quad f_{3}\right|_{F_{V} \gtrsim k_{e 3}}=\frac{F_{V} k_{e 3}}{F_{V}+k_{e 3}}=k_{e 3}$

This will lead to $\gamma \approx \gamma^{\prime}$, i.e. the reaction efficiency is not significantly altered with compartmentalization in comparison to the non-compartmentalized case.

Alternatively, when $F_{V} \ll k_{e 2}$ or $k_{e 3}$, we have

$\left.f_{2}\right|_{F_{V} \ll k_{e 2}}=\frac{F_{V} k_{e 2}}{F_{V}+k_{e 2}}=F_{V} \quad$ and $\left.\quad f_{3}\right|_{F_{V} \ll k_{e 3}}=\frac{F_{V} k_{e 3}}{F_{V}+k_{e 3}}=F_{V}$

This leads to

$\left.\gamma\right|_{F_{V} \ll k_{e 2} \& k_{e 3}}=\frac{k_{2} k_{3}}{\left(f_{2}+k_{2}\right)\left(f_{3}+k_{3}\right)}=\frac{k_{2} k_{3}}{\left(F_{V}+k_{2}\right)\left(F_{V}+k_{3}\right)}=\frac{1}{\left(\frac{F_{V}}{k_{2}}+1\right)\left(\frac{F_{V}}{k_{3}}+1\right)}$

$\left.T O F_{m=1}\right|_{F_{V} \ll k_{e 2} \& k_{e 3}}=\frac{k_{1} k_{2} k_{3} F_{V} C_{A}}{\left(k_{1} C_{A}-\frac{k_{1} k_{2} k_{3} C_{A}}{\left(F_{V}+k_{2}\right)\left(F_{V}+k_{3}\right)}+F_{V}\right)\left(F_{V}+k_{2}\right)\left(F_{V}+k_{3}\right)}$

The equations noted above suggest that optimal, near-unity reaction efficiency $\gamma$, high TOF, and low $R_{I}$ values would be obtained when $F_{V} \ll k_{2}$ and $k_{3}$, which is consistent with our observations in Figure 2.

Lastly, we explored $R_{I}$ and TOF as functions of $F_{V}$ and $k_{2}$ when accounting for $C_{C a t, t o t a l}$, (model $C_{\text {Cat,total }}$ ) as briefly discussed earlier, displayed in Supplementary Figures S5-S7, which shows little to no difference to the previous scenario without $C_{C a t, t o t a l}$. We do not explore $\gamma$ under model $C_{C a t, t o t a l}$, as $\gamma$ has no [Cat] dependence. For simplicity, we only explore the scenario accounting for $C_{\text {Cat,total }}$ when $\mathrm{m}=1$, though we provide general solutions when $\mathrm{m}=2$ in section S2A. Supplementary Figures S5A and S6A display $R_{I, m=1}$ as a function of $F_{V}$ and $k_{2}$, and $F_{V}$ alone for model $C_{\text {Cat,total }}$. In comparison to Figure $2 \mathrm{~B}$ and 3B, Figures S5A and S6A also predict that 
$R_{I}$ increases exponentially with $F_{V}$, and decreases with $k_{2}$, with the only difference being predicted values of $R_{I}$ when accounting for $C_{C a t, \text { total }}$. Supplementary Figures S5B and S6B display TOF $F_{m=1}$ as a function of $F_{V}$ and $k_{2}$, and $F_{V}$ alone in this alternate scenario. In comparison to plots in Figure $2 \mathrm{C}$ and $3 \mathrm{C}$, Figures S5B and 6B also predict TOF to decrease exponentially with $F_{V}$, and increase with $k_{2}$, with a difference in actual value of $T O F$ when accounting for $C_{\text {Cat,total }}$. Figure S7 displays $R_{I, m=1}$ and $T O F$ as a function of $k_{2}$ alone, compared to the corresponding non-compartmentalized metrics for model $C_{\text {Cat,total }}$ are also plotted. Similar to Figure 3B, compartmentalized $R_{I}$ (Figure $\mathrm{S} 7 \mathrm{~A}$ ) is predicted to be much smaller than non-compartmentalized $R_{I}$ at high $k_{2}$. However, at low $k_{2}$, it is predicted that a compartmentalized system will have greater $R_{I}$, when accounting for

A $\gamma\left(F_{v}\right)$

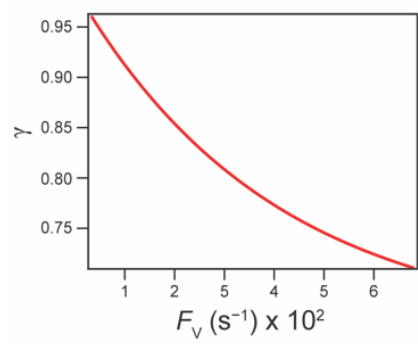

D $\gamma\left(k_{\mathrm{e} 2}, F_{v}\right)$

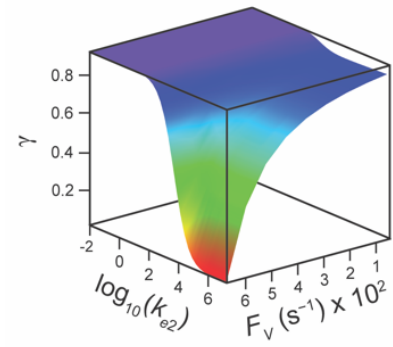

B $\log _{10}\left(R_{1, \mathrm{~m}=1}\left(F_{\mathrm{v}}\right)\right)$

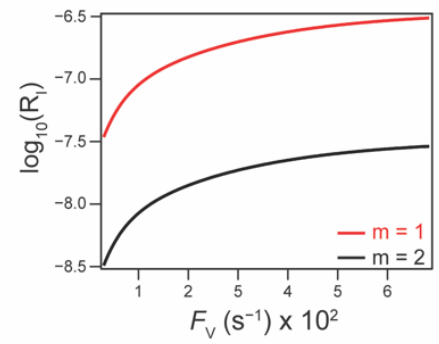

E $\log _{10}\left(R_{l, m=1}\left(k_{e 2}, F_{v}\right)\right)$

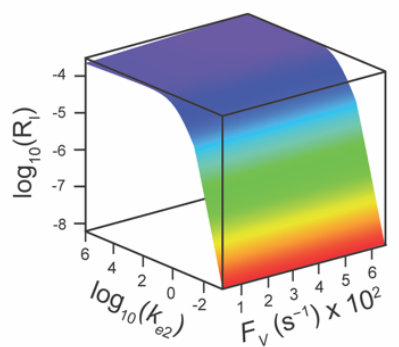

C $\log _{10}\left(\operatorname{TOF}_{\mathrm{m}=1}\left(F_{\mathrm{v}}\right)\right)$

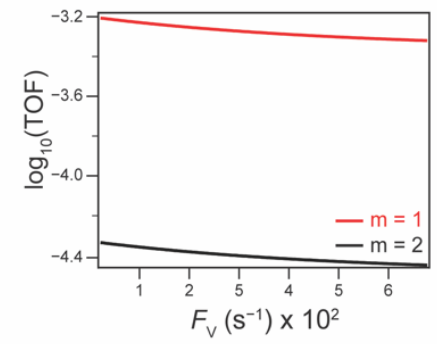

$\mathbf{F} \log _{10}\left(\mathrm{TOF}_{\mathrm{m}=1}\left(k_{e 2}, F_{\mathrm{v}}\right)\right)$

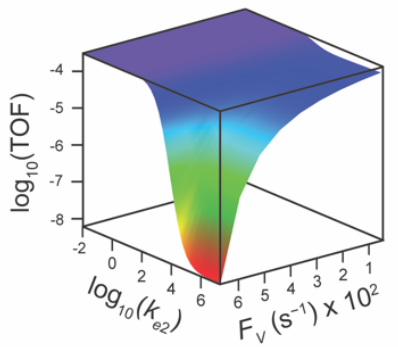

Figure 3. Graphical representations of compartmentalized reaction efficiency $(\gamma)$, rate of intermediate outflux $\left(R_{I}\right)$, and turnover frequency $(T O F)$ as a function of volumetric diffusive conductance $\left(F_{V}\right)$ alone $(\mathbf{A}-\mathbf{C})$ and as a function of $F_{V}$ and logarithm of the rate constant $\left(k_{e 2}\right)$ of Cat $-A$ deactivation $(\mathbf{D}-\mathbf{F})$. Kinetic parameters for panels $\mathbf{A}-\mathbf{F}$ are set at rate constant $\left(k_{1}\right)$ for oxidative addition $\left(k_{1}=0.1 \mathrm{M}^{-1} \mathrm{~s}^{-1}\right.$ for $\mathrm{m}=1$ and $\left.10 \mathrm{M}^{-2} \mathrm{~s}^{-1} \mathrm{~m}=2\right)$ (excluding $\gamma$ plots $\mathbf{A}$ and $\mathbf{D}$ ), rate constant for isomerization/migratory insertion (Iso/MI) $\left(k_{2}\right)$ and rate constant $\left(k_{e 2}\right)$ for Cat-A deactivation in competition with Iso/MI $\left(k_{2}=k_{e 2}=1 \times 10^{3} \mathrm{~s}^{-1}\right)$, and rate constant $\left(k_{3}\right)$ for reductive elimination and rate constant $\left(k_{e 3}\right)$ for $C a t-B$ deactivation $\left(k_{3}=k_{e 3}=1 \times 10^{6} \mathrm{~s}^{-1}\right)$. Plots $\mathbf{D}$ and $\mathbf{F}$ are rotated about the z-axis for clarity.

$C_{\text {Cat,total }}$, suggesting that compartmentalization may be marginally 
disadvantageous under certain parameters. In comparison with Figure 2F, Figure S7B shows that compartmentalized TOF also outcompetes non-compartmentalized as a function $k_{2}$ under model $C_{C a t, t o t a l}$, with a slight difference in actual value of TOF. Taken together, Figures S5-7 suggest that under the range of kinetic and diffusive parameters explored herein, approximating the initial catalyst concentration in bulk as constant (Figures 2-3, S1-4) does not significantly alter the conclusions of the model.

\section{Implication on the design of compartmentalized catalysis}

The established kinetic model and the numerical evaluation offers more affirmative answers to the efficacy of compartmentalization organometallic catalysis and, if needed, what is the desired properties of the established compartment. When the rate constants of the steps in the catalytic cycle $\left(k_{2}\right.$ and $\left.k_{3}\right)$ are commensurate with the rate constants of deactivation steps $\left(k_{e 2}\right.$ and $\left.k_{e 3}\right)$, i.e. $k_{2} \gtrsim k_{e 2}$ and $k_{3} \gtrsim k_{e 3}$, compartmentalization is not necessary since the intrinsic reactivity of catalysis is sufficiently fast with respect to side reactions. Compartmentalization should be considered when $k_{2}<k_{e 2}$ and $k_{3}<k_{e 3}$, when the intrinsic catalytic reactivity cannot outcompete the deactivation pathway. The efficacy of compartmentalization will be observable, as long as the compartment's volumetric diffusive conductance $F_{V}$ is much smaller than $k_{e 2}$ or $k_{e 3}$ $\left(F_{V} \ll k_{e 2}\right.$ or $\left.k_{e 3}\right)$. Nonetheless one interesting conclusion from our analysis is that maximal efficacy of compartmentalization (reaction efficiency $\gamma \rightarrow 1$ ) demands $F_{V}$ to be smaller not only than the rate constants of deactivation steps $\left(k_{e 2}\right.$ and $\left.k_{e 3}\right)$ but also than the rate constants of steps in the catalytic cycle $\left(k_{2}\right.$ and $\left.k_{3}\right)$. This requirement for maximal $\gamma$ stems from the fact that a "leaky" compartment with large $F_{V}$ is not sufficient to conserve the yielded intermediates and is prone to deactivation. Practically, such a requirement is indeed a blessing for organometallic chemistry. As typical organometallic studies do not commonly characterize the deactivating side reactions, there lacks detailed kinetic information for compartment design, as the values of $k_{e 2}$ or $k_{e 3}$ were needed to determine the range of desirable $F_{V}$ values. Though we posit that designing a confined catalytic cycle to have $F_{V}<k_{2}$ and $F_{V}<k_{3}$ is sufficient for a compartment to "revive" a proposed, unfunctional catalytic cycle, future design of compartmentalization can be simplified.

The feasibility of obtaining the range of $F_{V}$ from the kinetics of the proposed catalytic cycle offers more guidance for the materials design for the compartment. As $F_{V}$ equals the product of 
compartment boundary's permeability $(p)$ and its total surface area $(S A)$, while normalized by the volume $(V)$ of the corresponding compartment, ${ }^{26}$ multiple synthetic handles could be applied to achieve a desirable $F_{V}$ value. A less permeable interface at the boundary of compartment as well as smaller surface-to-volume ratio will help to reduce the mass transport hence the value of $F_{V}$. Characterization techniques that help determine encapsulation geometry and assess permeability, such as electron microscopies and chromatographic methods should be welcomed for more detailed mechanistic investigations in experimental demonstration. ${ }^{46-49}$ One interesting result from this argument is that a compartment of extremely small dimension, for example of nanoscopic scale, may not be necessarily beneficial, since nanoscopic dimensions can create an equivalently "leaky" compartment when normalized to the compartment volume. Careful design is recommended before experimental implementation.

Last, we cautioned that our established model only considers the mass transport of catalysts and assumes an unconditionally fast supply of substrate $A$ and quick removal of product $B$. While such assumptions have their real-life correspondence under certain circumstances (vide supra), the established model is incapable of accounting for the possible mass-transport limitation from substrate and products, which could be induced by a small $F_{V}$ value recommended by the model. Given that, we cautioned that a lower bound of $F_{V}$ exists for optimal performance in practical applications, and an unnecessarily small value of $F_{V}$ could be detrimental to the compartment design.

\section{CONCLUSION}

Here we have developed a kinetic framework for compartmentalizing organometallic catalysis, using a classical three step cycle consisting of OA, Iso/MI, and RE in that order. Under the same kinetic and diffusive parameters, the kinetic model predicts that key reaction metrics, derived from solving steady state equations of catalytic species, are significantly enhanced versus a homogenous counterpart. Furthermore, we demonstrated that careful design of a structured material to produce ideal volumetric diffusive conductance $\left(F_{V}\right)$ values in relation to kinetic parameters is a viable approach to optimization by plotting key reaction metrics as functions of $F_{V}$

and rate constants $k_{1}$ and $k_{2}$. From this, we conclude that confinement essentially induces a reaction to compete with influx and outflux instead of deactivation, provided deactivating media are adequately barred from the compartment. As diffusion into and out of a compartment can be 
tuned by confinement geometry, this offers a clear handle for optimization that freely diffusing systems do not possess. We also derived an additional kinetic framework accounting for both total catalyst concentration and catalyst in the bulk (model $\left.C_{C a t, t o t a l}\right)$, which ultimately yielded the same conclusions as when not accounting for total catalyst concentration. Lastly, we offered insight into accounting for various approaches to compartmentalization, where rigorous definition of confinement will be instrumental. The results from this study will assist in the a priori design of compartmentalized organometallics for enhanced catalytic performance. 


\section{ASSOCIATE CONTENT}

\section{Supporting Information}

The Supporting Information is available free of charge on the ACS Publications website at DOI: Mathematical derivation of compartmentalized and non-compartmentalized kinetic models, tables of solutions to key reaction metrics, and additional figures.

\section{Author Information}

C. L. supervised the project. B. J. J. performed the mathematical derivations for the theoretical framework. All the authors discussed the results and assisted during the manuscript preparation.

Corresponding Author

*Email: chongliu@chem.ucla.edu

ORCID

Brandon J. Jolly: 0000-0003-4991-5212

Chong Liu: 0000-0001-5546-3852

\section{Notes}

The authors declare no competing financial interest.

\section{ACKNOWLEDGEMENTS}

C. L. acknowledges the NSF Award (CHE-2027330), startup fund from the University of California, Los Angeles, and the financial support of the Jeffery and Helo Zink Endowed Professional Development Term Chair. 
TABLE OF CONTENTS

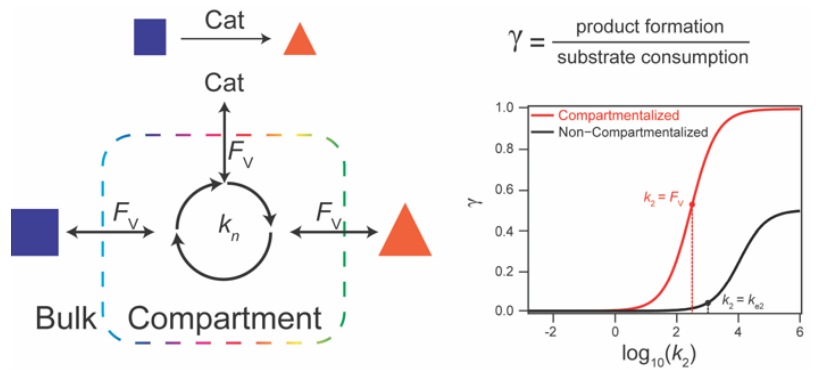




\section{REFERENCES}

1. Hurtley, S., Location, Location, Location. Science 2009, 326, 1205-1205.

2. Chen, A. H.; Silver, P. A., Designing Biological Compartmentalization. Trends Cell Biol. 2012, 22, 662-670.

3. Jandt, U.; You, C.; Zhang, Y. H. P.; Zeng, A. P., Compartmentalization and Metabolic Channeling for Multienzymatic Biosynthesis: Practical Strategies and Modeling Approaches. Adv. Biochem. Eng. Biotechnol. 2013, 137, 41-65.

4. Zecchin, A.; Stapor, P. C.; Goveia, J.; Carmeliet, P., Metabolic pathway compartmentalization: an underappreciated opportunity? Curr. Opin. Biotechnol. 2015, 34, 73-81. 5. Avalos, J. L.; Fink, G. R.; Stephanopoulos, G., Compartmentalization of Metabolic Pathways in Yeast Mitochondria Improves the Production of Branched-Chain Alcohols. Nat. Biotechnol. 2013, 31, 335-341.

6. Tu, B. P., Logic of the Yeast Metabolic Cycle: Temporal Compartmentalization of Cellular Processes. Science 2005, 310, 1152-1158.

7. Alam, M. T.; Olin-Sandoval, V.; Stincone, A.; Keller, M. A.; Zelezniak, A.; Luisi, B. F.; Ralser, M., The self-inhibitory nature of metabolic networks and its alleviation through compartmentalization. Nat. Commun. 2017, 8, 1-13.

8. Cannon, G. C.; Bradburne, C. E.; Aldrich, H. C.; Baker, S. H.; Heinhorst, S.; Shively, J. M., Microcompartments in Prokaryotes: Carboxysomes and Related Polyhedra. Appl. Environ. Microbiol. 2001, 67, 5351-5361.

9. $\quad$ Rae, B. D.; Long, B. M.; Whitehead, L. F.; Förster, B.; Badger, M. R.; Price, G. D., Cyanobacterial Carboxysomes: Microcompartments that Facilitate $\mathrm{CO}_{2}$ Fixation. J. Mol. Microbiol. Biotechnol. 2013, 23, 300-307.

10. Anderson, K. S.; Miles, E. W.; Johnson, K. A., Serine Modulates Substrate Channeling in Tryptophan Synthase. A Novel Intersubunit Triggering Mechanism. J. Biol. Chem. 1991, 266, 8020-8033.

11. Chavan, K. S.; Calabrese Barton, S., Simulation of Intermediate Channeling by Nanoscale Confinement. J. Phys. Chem. C 2018, 122, 14474-14480.

12. Jordan, P. C.; Patterson, D. P.; Saboda, K. N.; Edwards, E. J.; Miettinen, H. M.; Basu, G.; Thielges, M. C.; Douglas, T., Self-Assembling Biomolecular Catalysts for Hydrogen Production. Nat. Chem. 2016, 8, 179-185.

13. Chen, W.-H.; Vázquez-González, M.; Zoabi, A.; Abu-Reziq, R.; Willner, I., Biocatalytic Cascades Driven by Enzymes Encapsulated in Metal-Organic Framework Nanoparticles. Nat. Catal. 2018, 1, 689-695.

14. Vázquez-González, M.; Wang, C.; Willner, I., Biocatalytic Cascades Operating on Macromolecular Scaffolds and in Confined Environments. Nat. Catal. 2020, 3, 256-273.

15. Idan, O.; Hess, H., Origins of Activity Enhancement in Enzyme Cascades on Scaffolds. ACS Nano 2013, 7, 8658-8665.

16. Zhang, Y.; Hess, H., Toward Rational Design of High-efficiency Enzyme Cascades. ACS Catalysis 2017, 7, 6018-6027.

17. Natinsky, B. S.; Lu, S.; Copeland, E. D.; Quintana, J. C.; Liu, C., Solution Catalytic Cycle of Incompatible Steps for Ambient Air Oxidation of Methane to Methanol. ACS Cent. Sci. 2019, $5,1584-1590$.

18. Leenders, S. H. A. M.; Gramage-Doria, R.; De Bruin, B.; Reek, J. N. H., Transition Metal Catalysis in Confined Spaces. Chem. Soc. Rev. 2015, 44, 433-448. 
19. Lu, J.; Dimroth, J.; Weck, M., Compartmentalization of Incompatible Catalytic Transformations for Tandem Catalysis. J. Am. Chem. Soc. 2015, 137, 12984-12989.

20. Rayder, T. M.; Bensalah, A. T.; Li, B.; Byers, J. A.; Tsung, C.-K., Engineering Second Sphere Interactions in a Host-Guest Multicomponent Catalyst System for the Hydrogenation of Carbon Dioxide to Methanol. J. Am. Chem. Soc. 2021, 143, 1630-1640.

21. Simms, R. W.; Cunningham, M. F., Compartmentalization of Reverse Atom Transfer Radical Polymerization in Miniemulsion. Macromolecules 2008, 41, 5148-5155.

22. Smeets, N. M. B.; Heuts, J. P. A.; Meuldijk, J.; Cunningham, M. F.; Van Herk, A. M., Evidence of Compartmentalization in Catalytic Chain Transfer Mediated Emulsion Polymerization of Methyl Methacrylate. Macromolecules 2009, 42, 7332-7341.

23. Wang, K.; Jordan, J. H.; Hu, X. Y.; Wang, L., Supramolecular Strategies for Controlling Reactivity within Confined Nanospaces. Angew. Chem. Int. Ed. 2020, 59, 13712-13721.

24. Wayland, B. B.; Ba, S.; Sherry, A. E., Activation of Methane and Toluene by Rhodium(II) Porphyrin Complexes. J. Am. Chem. Soc. 1991, 113, 5305-5311.

25. Hinzpeter, F.; Gerland, U.; Tostevin, F., Optimal Compartmentalization Strategies for Metabolic Microcompartments. Biophysical Journal 2017, 112, 767-779.

26. Tsitkov, S.; Hess, H., Design Principles for a Compartmentalized Enzyme Cascade Reaction. ACS Catalysis 2019, 9, 2432-2439.

27. Berg, H. C., Random Walks in Biology. Princeton University Press: 1993.

28. Miessler, G.; Tarr, D., Inorganic Chemistry. Upper Saddle River, NJ: Pearson Education Inc.: 2004.

29. Labinger, J. A., Tutorial on Oxidative Addition. Organometallics 2015, 34, 4784-4795.

30. Breno, K. L.; Pluth, M. D.; Landorf, C. W.; Tyler, D. R., Aqueous Phase Organometallic Catalysis Using $(\mathrm{MeCp})_{2} \mathrm{Mo}(\mathrm{OH})\left(\mathrm{H}_{2} \mathrm{O}\right)^{+}$. Intramolecular Attack of Hydroxide on Organic Substrates. Organometallics 2004, 23, 1738-1746.

31. Ellis, P. R.; Pearson, J. M.; Haynes, A.; Adams, H.; Bailey, N. A.; Maitlis, P. M., Oxidative Addition of Alkyl Halides to Rhodium(I) and Iridium(I) Dicarbonyl Diiodides: Key Reactions in the Catalytic Carbonylation of Alcohols. Organometallics 1994, 13, 3215-3226.

32. Espenson, J. H.; Pestovsky, O.; Huston, P.; Staudt, S., Organometallic Catalysis in Aqueous Solution: Oxygen Transfer to Bromide. Journal of the American Chemical Society 1994, 116, 2869-2877.

33. Ju, T. D.; Lang, R. F.; Roper, G. C.; Hoff, C. D., Oxidative Addition of Butanethiol and Thiophenol to the $\cdot \mathrm{Cr}(\mathrm{CO})_{3} \mathrm{C}_{5} \mathrm{Me}_{5}$ Radical. Kinetic and Thermodynamic Study of a Third-Order Reaction and Its Catalysis. J. Am. Chem. Soc. 1996, 118, 5328-5329.

34. Heiden, Z. M.; Rauchfuss, T. B., Homogeneous Catalytic Reduction of Dioxygen Using Transfer Hydrogenation Catalysts. J. Am. Chem. Soc. 2007, 129, 14303-14310.

35. Stein, W., Transport and diffusion across cell membranes. Elsevier Inc.: 1986.

36. Astruc, D., Organometallic chemistry and catalysis. Springer: 2007.

37. Ford, P. C.; Massick, S., Time Resolved Spectroscopic Studies Relevant to Reactive Intermediates in Homogeneous Catalysis. The Migratory Insertion Reaction. Coord. Chem. Rev. 2002, 226, 39-49.

38. Massick, S. M.; Ford, P. C., Use of a High-Pressure/Variable-Temperature Infrared Flow Cell To Examine the Reaction Kinetics of the Migratory Insertion Intermediate $\left(\eta^{5}\right.$ $\left.\mathrm{C}_{5} \mathrm{H}_{5}\right) \mathrm{Fe}(\mathrm{CO}) \mathrm{C}(\mathrm{O}) \mathrm{CH}_{3}$ by Time-Resolved Spectroscopy. Organometallics 1999, 18, 4362-4366.

39. Nilsson, P.; Wendt, O. F., Kinetic Investigation of a $\mathrm{PC}\left(s p^{3}\right) \mathrm{P}$ pincer palladium (II) complex in the Heck reaction. J. Organomet. Chem. 2005, 690, 4197-4202. 
40. Jin, L.; Zhang, H.; Li, P.; Sowa Jr, J. R.; Lei, A., What is the Rate of the Csp ${ }^{2}-$ Csp $^{2}$ Reductive Elimination Step? Revealing an Unusually Fast Ni-Catalyzed Negishi-Type Oxidative Coupling Reaction. J. Am. Chem. Soc. 2009, 131, 9892-9893.

41. Pregosin, P. S.; Kumar, P. G. A.; Fernández, I., Pulsed Gradient Spin-Echo (PGSE) Diffusion and ${ }^{1} \mathrm{H},{ }^{19} \mathrm{~F}$ Heteronuclear Overhauser Spectroscopy (HOESY) NMR Methods in Inorganic and Organometallic Chemistry: Something Old and Something New. Chem. Rev. 2005, 105, 2977-2998.

42. Drago, D.; Pregosin, P. S.; Pfaltz, A., Selective anion effects in chiral complexes of iridium via diffusion and HOESY data: relevance to catalysis. Chem. Commun. 2002, 286-287.

43. Natinsky, B. S.; Jolly, B. J.; Dumas, D. M.; Liu, C., Efficacy analysis of compartmentalization for ambient $\mathrm{CH}_{4}$ activation mediated by a $\mathrm{Rh}^{\mathrm{II}}$ metalloradical in a nanowire array electrode. Chem. Sci. 2021, 12, 1818-1825.

44. Bender, T. A.; Morimoto, M.; Bergman, R. G.; Raymond, K. N.; Toste, F. D., Supramolecular Host-Selective Activation of Iodoarenes by Encapsulated Organometallics. J. Am. Chem. Soc. 2019, 141, 1701-1706.

45. Qu, P.; Kuepfert, M.; Hashmi, M.; Weck, M., Compartmentalization and Photoregulating Pathways for Incompatible Tandem Catalysis. J. Am. Chem. Soc. 2021, 143, 4705-4713.

46. Huang, C.; Liu, C.; Chen, X.; Xue, Z.; Liu, K.; Qiao, X.; Li, X.; Lu, Z.; Zhang, L.; Lin, Z.; Wang, T., A Metal-Organic Framework Nanosheet-Assembled Frame Film with High Permeability and Stability. Adv. Sci. 2020, 7, 1903180.

47. Li, W.; Zhang, Y.; Xu, Z.; Meng, Q.; Fan, Z.; Ye, S.; Zhang, G., Assembly of MOF Microcapsules with Size-Selective Permeability on Cell Walls. Angew. Chem. Int. Ed. 2016, 55, 955-9.

48. Lei, L.; Zhang, Q.; Shi, S.; Zhu, S., Breathable Microgel Colloidosome: Gas-Switchable Microcapsules with $\mathrm{O}_{2}$ and $\mathrm{CO}_{2}$ Tunable Shell Permeability for Hierarchical Size-Selective Control Release. Langmuir 2017, 33, 6108-6115.

49. Haldoupis, E.; Watanabe, T.; Nair, S.; Sholl, D. S., Quantifying Large Effects of Framework Flexibility on Diffusion in MOFs: $\mathrm{CH}_{4}$ and $\mathrm{CO}_{2}$ in ZIF-8. ChemPhysChem 2012, 13, 3449-3452. 